\title{
A Scalable and Reliable Message Transport Service for the ATLAS Trigger and Data Acquisition System
}

\author{
Andrei Kazarov, Mihai Caprini, Serguei Kolos, Giovanna Lehmann Miotto and Igor Soloviev
}

\begin{abstract}
The ATLAS Trigger and Data Acquisition (TDAQ) is a large distributed computing system composed of several thousands of interconnected computers and tens of thousands applications. During a run, TDAQ applications produce a lot of control and information messages with variable rates, addressed to TDAQ operators or to other applications. Reliable, fast and accurate delivery of the messages is important for the functioning of the whole TDAQ system. The Message Transport Service (MTS) provides facilities for the reliable transport, the filtering and the routing of the messages, based on the publish-subscribe-notify communication pattern with content-based message filtering.

During the ongoing LHC shutdown, MTS was re-implemented, taking into account important requirements like reliability, scalability and performance, handling of slow subscribers case and also simplicity of the design and the implementation.

MTS uses CORBA middleware, a common layer for TDAQ infrastructure, and provides sending/subscribing APIs in the Java and $\mathrm{C}++$ programming languages.

The paper presents the design and the implementation details of MTS, as well as the results of performance and scalability tests executed on a computing farm with an amount of workers and working conditions which reproduced a realistic TDAQ environment during ATLAS operations.
\end{abstract}

\section{INTRODUCTION. MESSAGE REPORTING IN TDAQ.}

$\mathbf{T}$ HE ATLAS experiment at LHC is operated by the Trigger and Data Acquisition System (TDAQ) which is a complex distributed computing system composed by $O\left(10^{4}\right)$ of applications running on a farm of more then 2000 multicore computing nodes [1]. During data-taking runs TDAQ applications send out control and information messages which are addressed to other applications and TDAQ operators. Messages are reported by the applications using the ERS (Error Reporting System [2]) framework which enables messages to be defined, created and reported in user's code, and message routing to be configured at run-time. The main ERS output stream is the Message Transport Service (MTS), a TDAQ software core service which provides facilities for the reliable transport, the filtering and the routing of the messages, based on the publish-subscribe-notify communication pattern with

Manuscript received May 22, 2014.

A. Kazarov is with CERN,Geneva, Switzerland, on leave from Petersburg Nuclear Physics Institute, Kurchatov NPI, Gatchina, Russian Federation (email: Andrei.Kazarov@cern.ch).

M. Caprini is with NIPNE-HH, Bucharest, Romania (e-mail: Mihai.Caprini@cern.ch)

S. Kolos is with UCI, Irvine, CA, USA (e-mail: Serguei.Kolos@cern.ch).

G. Lehmann Miotto is with CERN, Geneva, Switzerland (e-mail: Giovanna.Lehmann@cern.ch).

I. Soloviev is with UCI, Irvine, CA, USA (e-mail: Igor.Soloviev@cern.ch). content-based message filtering. MTS allows other TDAQ applications (receivers) to subscribe to certain messages or to certain types of messages using combinations of ERS message attributes (e.g. message ID or severity) as subscription (i.e. filtering) criteria.

\section{MotiVATIONS AND REQUiREMENTS FOR MTS RE-IMPLEMENTATION}

During LHC shutdown, the MTS service was reimplemented, after a review of the user requirements. The most important requirements, which had strong impact on the design of MTS are discussed below:

\section{A. Performance and Scalability}

With appearing of the new multi-core processor architectures in the next generation of the TDAQ system, it is clear that the number of TDAQ applications (which are MTS senders) will increase, together with the possible peak rates of the reported messages. The MTS architecture shall be scalable with respect to the number of senders $\left(O\left(10^{4}\right)\right)$ and receivers $\left(O\left(10^{2}\right)\right.$ ) and shall be able to handle high message rates (few $k H z$ of reported messages), providing more throughput when needed by adding more working elements to the system and by utilizing all available CPU cores on worker nodes.

\section{B. Reliability}

Messages must still be delivered in case a part of the MTS infrastructure (e.g. a worker node or worker process) fails or becomes unreachable (a loss of messages which are buffered in a worker at the moment of a sudden crash is tolerated). There shall not be single point of failure in the MTS architecture, though MTS may rely on external TDAQ services. Also, MTS shall not cause blocking of user threads on the sender side (e.g. in case of unavailability of parts of MTS infrastructure).

\section{Handling of "slow" receivers}

MTS shall guarantee a "fair" receiver notification policy: the internal allocation of resources (e.g. worker delivery threads) must be such that, in cases when some slow (or even fully blocked) receivers are present in the system, this does not cause delays in delivery of messages to other receivers. Messages addressed to slow receivers must be buffered and eventually be delivered. 


\section{Simplicity}

The MTS implementation should use the available thirdparty toolkits, libraries and other TDAQ services whenever possible, thus making the code simpler and easier to maintain during the long life span of the ATLAS experiment.

\section{HIGH-LEVEL DESIGN}

MTS implements a publish-subscribe-notify communication pattern with content-based message filtering, where senders and receivers are linked indirectly: senders do not directly send messages to receivers. Instead they publish messages to MTS nodes (workers) and receivers subscribe in MTS for particular types of messages and then asynchronously get notified by MTS as soon as a message matching the subscription criteria arrives to the system.

The MTS infrastructure consists of a few identical workers, whose tasks are to share and balance the load and to provide fault-tolerant service in case of a failure of a particular worker process or its host. The worker is selected by senders in a random manner, which guarantees equal sharing of load among all workers (assuming that workers run on dedicated and identical nodes). Workers can be started and restarted independently, and new workers can be added even during a run if necessary.

Runtime information (the list of active workers and the list of active subscriptions) is shared within MTS using other common TDAQ services, namely the TDAQ naming service (IPC) and information sharing service (IS) [3]. The composition of the MTS package, collaboration diagram and interfaces provided by MTS are presented in Fig. 1

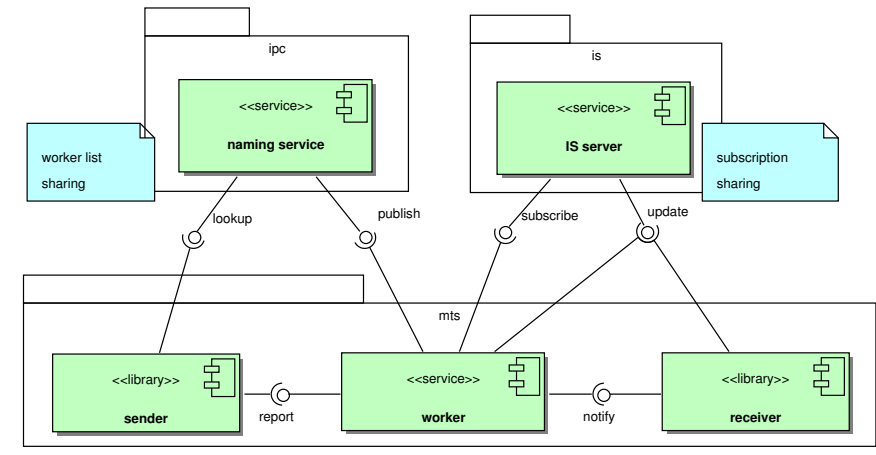

Fig. 1. MTS interfaces, components and collaboration with external packages

\section{IMPLEMENTATION DETAILS}

\section{A. IDL interfaces and use of CORBA}

The details of TCP/IP network communication between MTS actors (senders, workers and receivers) are hidden by the use of the CORBA protocol, which is a cross-platform communication middleware used for all TDAQ core services. Each actor defines and implements IDL (Interface Definition Language) interfaces, providing the declared functionality, and IDL methods are called remotely via CORBA by other actors. The IDL of MTS is very simple:

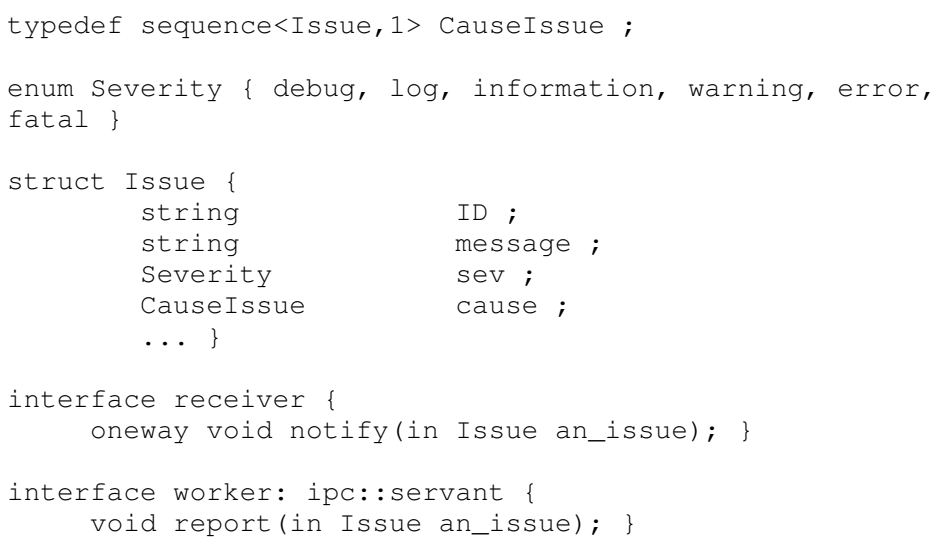

The CORBA method 'report' (from sender to worker) is declared two-way, in order to either reliably (with explicit confirmation) deliver the message to a worker or to get a fast notification (timeout) indicating that that particular worker is not available and to be able to retransmit the message to another worker. The CORBA timeout for 'report' call is set to a low value of $1 \mathrm{~s}$, in order not to block the sender thread for a long time.

The CORBA method 'notify' (from worker to receiver) is declared as one-way, which means that sending of messages is non-blocking (unless there is no space left in TCP buffers in case of slow receiver), and there is no wasting of time in notification threads.

The implementation of the MTS IDL is available in C++ and Java languages.

\section{B. Worker architecture and handling of slow subscriber case}

Receiving and sending of messages are decoupled in the worker, i.e. a thread which receives a message from a sender does not handle notification of receivers, instead it copies the message in a buffer (a queue). This is done in order to release the receiver thread as soon as possible and not to block the sender code. The worker includes two pools of threads and a number of buffers, as shown in Fig. 2. The first pool is a pool of message receiving threads (managed by CORBA), which performs the CPU-intensive job of matching all the subscriptions to the received message attributes and copying the matched messages to a dedicated buffer queue, allocated for each receiver. The other pool is a pool of delivery threads, which loops through the queues of not yet delivered messages and calls the 'notify' method on the receivers.

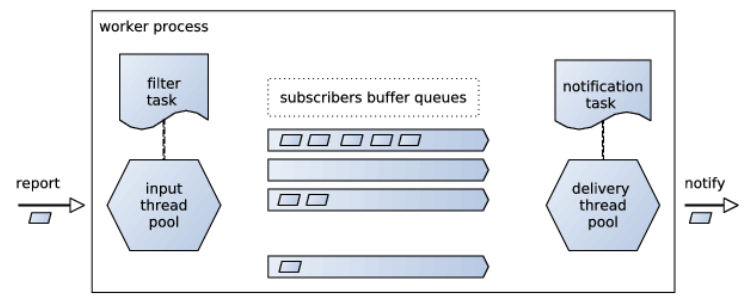

Fig. 2. Worker architecture 
Each thread pool is managed by a scheduler which assigns a task to a thread when a free thread becomes available. The scheduler for the input thread pool is a simple round-robin scheduler. The scheduler for the notification pool implements a "fair" algorithm whose main purpose is to avoid delays in notification of subscribers. This may happen if there are some slow subscribers in the system and all or most of the delivery threads gets busy with serving those slow subscribers. The fair scheduler assigns priorities to subscribers, taking into account the number of threads already allocated and the average delays in the 'notify' call to a subscriber. The slow subscribers get lower priority and therefore less resources from the notification pool. This guarantees the availability of resources for other subscribers.

For simplicity, no dropping of messages is implemented in a worker and the buffer queues are unlimited. Given the average size of messages and known number of messages sent in the system during a data-taking run (maximum is 2 millions of messages per run), it is assumed that MTS workers (running on modern 64bit hardware) can buffer all the messages sent during a run.

\section{Subscription criteria syntax with EBNF}

The selection criteria syntax enables filtering of messages using ERS message attributes: severity (sev), applicationID (app), messageID (msg), qualifiers (qual), grouping of expressions and arbitrary logical combination (and, or, not) of them. The syntax is defined using EBNF [4] notation:

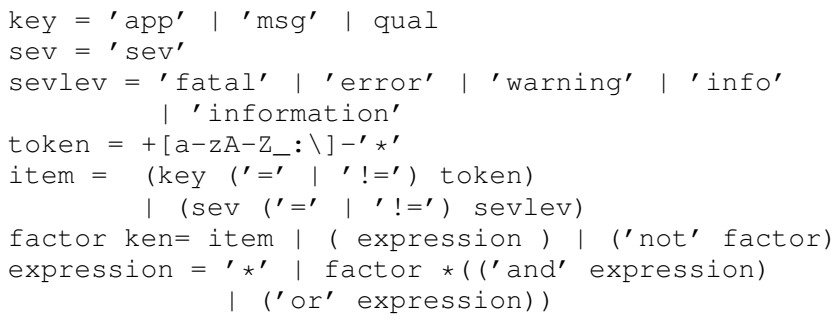

A typical example of a selection criteria is

( $\mathrm{sev}=\mathrm{ERROR}$ or sev=FATAL) or

(app=Tile* and not qual=debug)

The use of standard EBNF notation enabled the development of a very compact and maintainable implementation of the message filtering code which is based on the Boost::spirit framework [5]. The formal syntax listed above is translated almost literally to $\mathrm{C}++$ constructs in the code, and this is all that needs to be done to implement the matching and filtering in the worker library.

\section{RESULTS FROM SCALABILITY TESTS AND FROM FIRST RUNS IN 2014}

Table I summarizes the scalability measurements of MTS performed on a test bed (a cluster of 100 nodes) in conditions which reproduced a realistic TDAQ environment during ATLAS operations. The number of senders (3000-30000, each sending 1 message per second) and receivers (8-96) was varied. This resulted in total rates of reported messages to MTS system from $3 \mathrm{kHz}$ to $30 \mathrm{kHz}$ and total output rates (the sum of the rates of messages delivered from all workers to all receivers) up to $880 \mathrm{kHz}$. This rates was handled by 2 or 4 workers, each running 16 input and 16 output threads. The presence of buffering in workers or in senders (which results in latencies higher then normal) and the output bandwidth from a worker node was also measured, in order to understand the conditions where the system becomes saturated.

TABLE I

SUMMARY OF MTS SCALABILITY MEASUREMENTS ON TEST BED

\begin{tabular}{|l|l|l|l|l|l|l|}
\hline $\begin{array}{l}\text { \# of } \\
\text { senders } \\
\left(\begin{array}{l}1 \mathrm{~Hz} \\
\text { each) }\end{array}\right.\end{array}$ & $\begin{array}{l}\text { \# of } \\
\text { re- } \\
\text { ceivers }\end{array}$ & $\begin{array}{l}\text { \# of } \\
\text { work- } \\
\text { ers }\end{array}$ & $\begin{array}{l}\text { total } \\
\text { input } \\
\text { rate, } \\
\mathrm{kHz}\end{array}$ & $\begin{array}{l}\text { total } \\
\text { output } \\
\text { rate, } \\
\mathrm{kHz}\end{array}$ & $\begin{array}{l}\text { output } \\
\text { band- } \\
\text { width } \\
\text { from } \\
\text { single } \\
\text { worker, } \\
\text { MBps }\end{array}$ & remarks \\
\hline \hline 3000 & 80 & 2 & 3 & 285 & 50 & \\
\hline 3000 & 96 & 2 & 2.9 & 237 & 70 & buffering \\
\hline 15000 & 32 & 2 & 14.6 & 460 & 119 & $\begin{array}{l}\text { network } \\
\text { satura- } \\
\text { tion }\end{array}$ \\
\hline 15000 & 32 & 4 & 15 & 470 & 57 & buffering \\
\hline 15000 & 48 & 4 & 15 & 680 & 90 & $\begin{array}{l}\text { network } \\
\text { satura- } \\
\text { tion }\end{array}$ \\
\hline 30000 & 16 & 2 & 30 & 460 & 114 & \\
\hline 30000 & 32 & 4 & 30 & 880 & 77 & \\
\hline
\end{tabular}

The tests demonstrated that a single MTS worker can handle up to $15 \mathrm{kHz}$ of input and $230 \mathrm{kHz}$ of output messages rate, in this conditions saturating the $1 \mathrm{Gbps}$ network output link. A total number of 30k simultaneous senders can be handled by 2 workers running on 16 core nodes, and more workers can be added to scale the performance.

The plot in Fig 3 shows measurements of output rates and message delivery latency (time registered on receiver w.r.t. time of the message creation) as a function of input rate in tests done on a cluster of 130 nodes with 2 workers. It also demonstrates that MTS can saturate its network output link (1 Gbps), while keeping the average message delivery latency below 10 milliseconds.

MTS was used in production during milestone and technical runs of the TDAQ system in 2014. The infrastructure included 4 running workers. There were around 60000 applications running in the system and sending messages. Fig. 4 shows the rates (maximum and average) of the messages reported in MTS system during few days of running, which was of the order of few $k H z$ with maximum of $18 k \mathrm{~Hz}$.

\section{CONCLUSIONS}

A reliable, high-performing and scalable message transfer service is implemented for applications in the ATLAS TDAQ system for the LHC Run II. Functionality and performance of the service were validated during TDAQ technical and first ATLAS milestone runs in 2014.

Wide usage of other TDAQ services and third-party software (like CORBA and Boost) allowed the MTS implementation to 


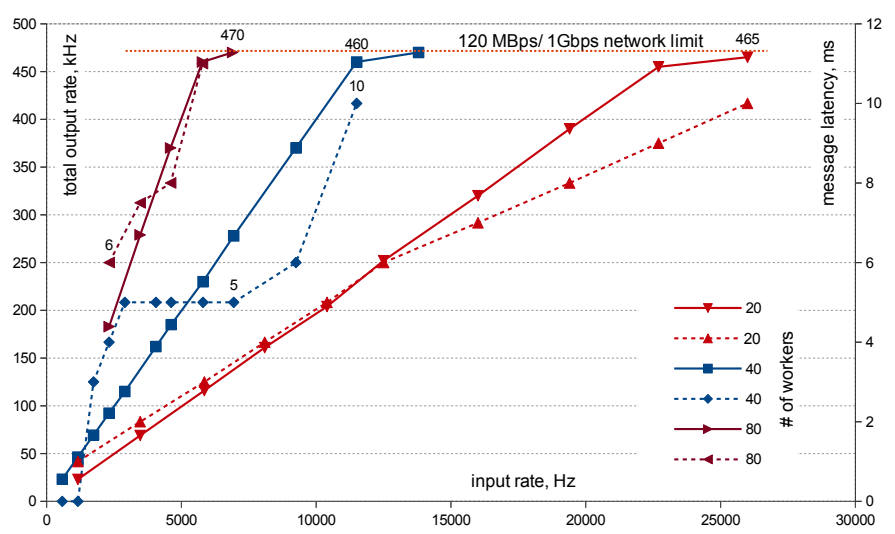

Fig. 3. Output rate (solid lines) and message latency (dashed lines) as function of input rate and number of receivers, for 20, 40 and 80 receivers

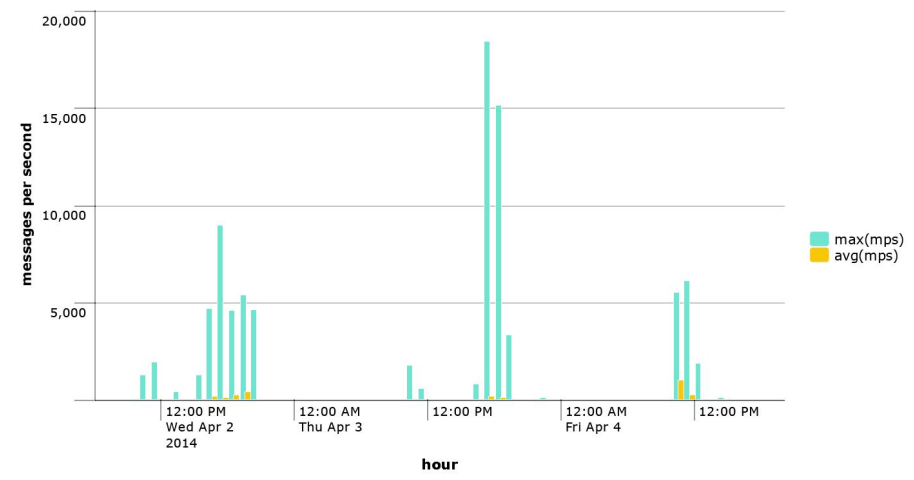

Fig. 4. Rates of messages reported in MTS in technical runs in April 2014. Maximum and average values in 1 hour slots are presented.

be compact and easy to maintain. The total size of the package is just 2300 lines of $\mathrm{C}++$ and 520 lines of Java code.

\section{REFERENCES}

[1] ATLAS Collaboration. Atlas high-level trigger, data acquisition and controls technical design report. Available: http://cdsweb.cern.ch/record/616089/, Jun 2003.

[2] A. dos Anjos, H.P. Beck, S. Kolos, B. Gorini, M. Wiesmann Error Handling and Error Reporting in TDAQ Applications Available: https://edms.cern.ch/file/459790/1.0/ErrorHandling.pdf

[3] Kolos, S. ; Boutsioukis, G. ; Hauser, R. High-performance scalable information service for the ATLAS experiment Real Time Conference (RT), 2012 18th IEEE-NPSS

[4] Richard E. Pattis, EBNF notation. Available: http://www.cs.cmu.edu/ pattis/misc/ebnf.pdf.

[5] Joel de Guzman, Hartmut Kaiser. Boost Spirit documentation. Available: http://www.boost.org/doc/libs/1_55_0/libs/spirit/doc/html/index.html 